\title{
REVIEW
}

\section{Arrhythmias in cardiac sarcoidosis}

\author{
Alexandru B. Chicos'
}

\section{INTRODUCTION}

Cardiac sarcoidosis (CS) is an under-diagnosed and under-treated etiology of cardiac arrhythmias and sudden death. This article reviews, using a series of case vignettes, some of the clinical presentations in cardiology and the challenges in diagnosis and treatment, particularly focused on arrhythmia.

\section{EPIDEMIOLOGY AND ARGUMENT FOR RELEVANCE IN ROMANIA}

Cardiac sarcoidosis is a rare disease. However, there may be an opportunity to identify and help a significant number of patients that go undiagnosed and untreated. Cardiologists and cardiac electrophysiologists are uniquely positioned to diagnose some of the sarcoidosis patients that are at the highest risk of morbidity and mortality - those with cardiac involvement.

Sarcoidosis is difficult to diagnose and can be clinically silent. It is a systemic disease and can present in a multitude of ways, reason for which it is considered to be one of the "great imitators". Patients can present to any medical subspecialty: pulmonology, infectious disease, rheumatology, ophthalmology, dermatology, neurology, oncology, cardiology, etc. Diagnosis is even more difficult when other respiratory conditions, such as pulmonary tuberculosis, that can have similar clinical presentation, are prevalent ${ }^{1,2}$.

The prevalence of sarcoidosis has been estimated between 4.7-64 per 100.000, with highest rates reported in Northern European and African American individuals, and in women. A I5-year European study ${ }^{3}$ from 1984 found an annual incidence of sarcoidosis (in the population older than 15 years) of 19/100,000. In this report, the accumulated lifetime risk of sarcoido-

\footnotetext{
' Department of Cardiac Electrophysiology, Bluhm Cardiovascular Institute, Feinberg School of Medicine, Northwestern University, Chicago, USA.
}

sis was $\mathrm{I} .3 \%$ for women and almost $\mathrm{I} \%$ for men. However, the disease is encountered worldwide and is probably more prevalent than thought, including in Central and Eastern Europe. Deubelbeiss et $\mathrm{al}^{4}$ found a prevalence of sarcoidosis in Switzerland that was higher than assumed based on previous international estimates. The prevalence of lifetime and currently active sarcoidosis were $12 \mathrm{I}$ and 44 per 100.000 inhabitants, respectively. The mean annual incidence of sarcoidosis was $7 / 100.000$. An Italian study ${ }^{5}$ found a prevalence of sarcoidosis estimated at 48/100,000 in the Parma region for the years 2010-2013, much higher than previously thought, and as high as 196/100,000 in some areas. A study ${ }^{6}$ in Columbus, Ohio, found an unexpectedly high prevalence, at least 50/100,000 and possibly as high as $200 / 100.000$, in population that was predominantly Causian (White, 74\%). In the Romanian Registry for Interstitial Lung Diseases (REGIS) ${ }^{1,2}$, I44 patients were enrolled over 3 years, of which 28 were diagnosed with sarcoidosis, most with mediastinal and/ or pulmonary involvement. Authors concluded that, in Romania, interstitial lung diseases, including sarcoidosis, are under-diagnosed and under-reported, and that diagnosis is often made in advanced stages of disease despite availability of diagnostic tools, such as bronchoalveolar lavage and biopsy, in dedicated centers.

Cardiac sarcoidosis is under-diagnosed everywhere in the world. Myocardial involvement can be clinically silent for years. Diagnosis is difficult even in patients presenting with cardiac symptoms. Patients who eventually are diagnosed with cardiac sarcoidosis experience a significant delay in definitive diagnosis, often for years. Among 275 patients enrolled in an international cardiac sarcoidosis registry in 21 centers, mostly in US and Japan, showed that the time from cardiac symp-

\footnotetext{
$\checkmark$ Contact address:

Alexandru B. Chicos, MD

Associate Professor, Cardiac Electrophysiology, Bluhm Cardiovascular Institute, Feinberg School of Medicine, Northwestern University, Chicago, USA.

E-mail: achicos@nm.org
} 
tom onset to diagnosis of cardiac sarcoidosis was 16.1 +/- 34 months $^{7}$. Access to advanced cardiac imaging such as MRI and FDG-PET is one limitation. Assuming a prevalence of currently active cases of sarcoidosis in Romania of $10-50 / 100.000$, this would translate to $\sim 2.000$ to 10.000 patients with active sarcoidosis. An estimated $5 \%$ of them could have manifest cardiac involvement ( $100-500$ patients) and up to $50 \%$ (up to I.000-5.000 patients) may have apparently asymptomatic cardiac involvement. It is therefore essential to screen for cardiac involvement if a diagnosis of sarcoidosis involving any organ system is made, and also to consider and look for sarcoidosis if the patient presents with unexplained cardiac symptoms or findings. This is particularly important because cardiac sarcoidosis is treatable, but, if left untreated, it can lead to significant morbidity and death from arrhythmia or progressive myocardial damage.

\section{CLINICAL MANIFESTATIONS AND DIAGNOSIS}

Sarcoidosis is a systemic disease, and can involve any organ system. It can present with a broad spectrum of symptoms, or it can be asymptomatic and diagnosed incidentally ${ }^{8}$. Its cardiac manifestations depend on the location, extent and degree of inflammation, and include conduction abnormalities, ventricular arrhythmia and heart failure. Figure I illustrates this in a patientfriendly schematic representation. For the diagnosis of cardiac sarcoidosis, it is important to have keep a high index of suspicion and try as much as possible to obtain tissue diagnosis. It is difficult and potentially detrimental to embark on long-term immunosuppression without confidence in the diagnosis. Diagnostic sets of criteria have been proposed by the Japanese Circulation Society (JCS) ${ }^{8}$, Heart Rhythm Society (HRS) ${ }^{9}$, and the World Association for Sarcoidosis and Other Granulomatous Disorders (WASOG) (Table I and Table 2).

The majority of cases are diagnosed by typical cardiac involvement combined with extra-cardiac biopsy. Cardiac biopsy has a relatively low-yield, but can be useful in selected patients. Tissue diagnosis should always be considered. Other causes of non-caseating granulomas should be excluded. There are some situations when the pattern of involvement is very characteristic, for example bilateral hilar lymphadenopathy with increased FDG-PET uptake in the lymph nodes as well as patchy myocardial uptake. These are exceptions when, if tissue diagnosis cannot be obtained, a presumptive diagnosis of cardiac sarcoidosis can be made. The updated Japanese criteria ${ }^{8}$ allow for clinical diagnosis, in selected situations, in the absence of tissue diagnosis.

\section{PATHOPHYSIOLOGY OF ARRHYTHMIAS IN CARDIAC SARCOIDOSIS}

The pathophysiology of arrhythmias in cardiac sarcoidosis is related to the inflammatory infiltrates. „Early lesions” consist of lymphocytic myocarditis. Lesions containing granulomas (non-necrotizing) along with lymphocytic myocarditis have been termed ,intermediate". In the chronic stage, areas of active inflammation have caused myocyte loss and repair fibrosis, and

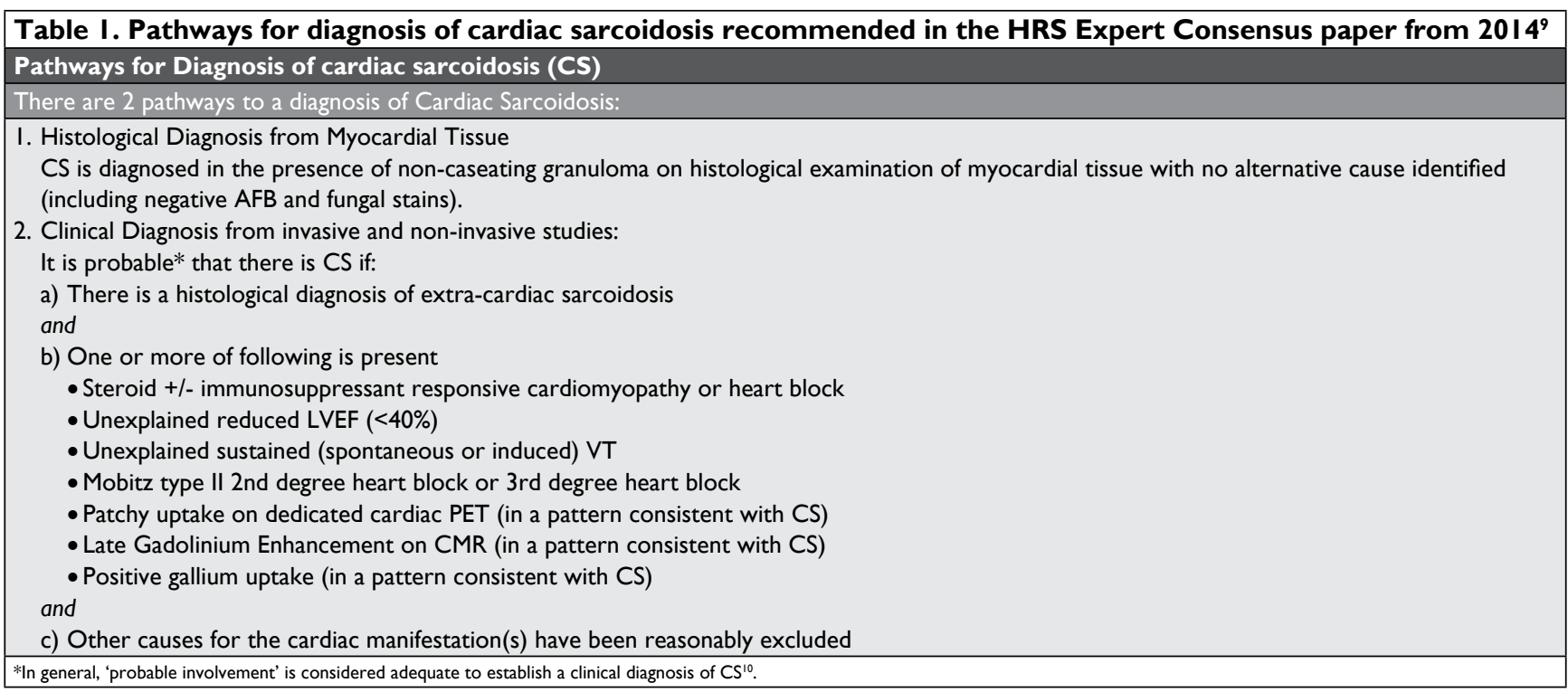




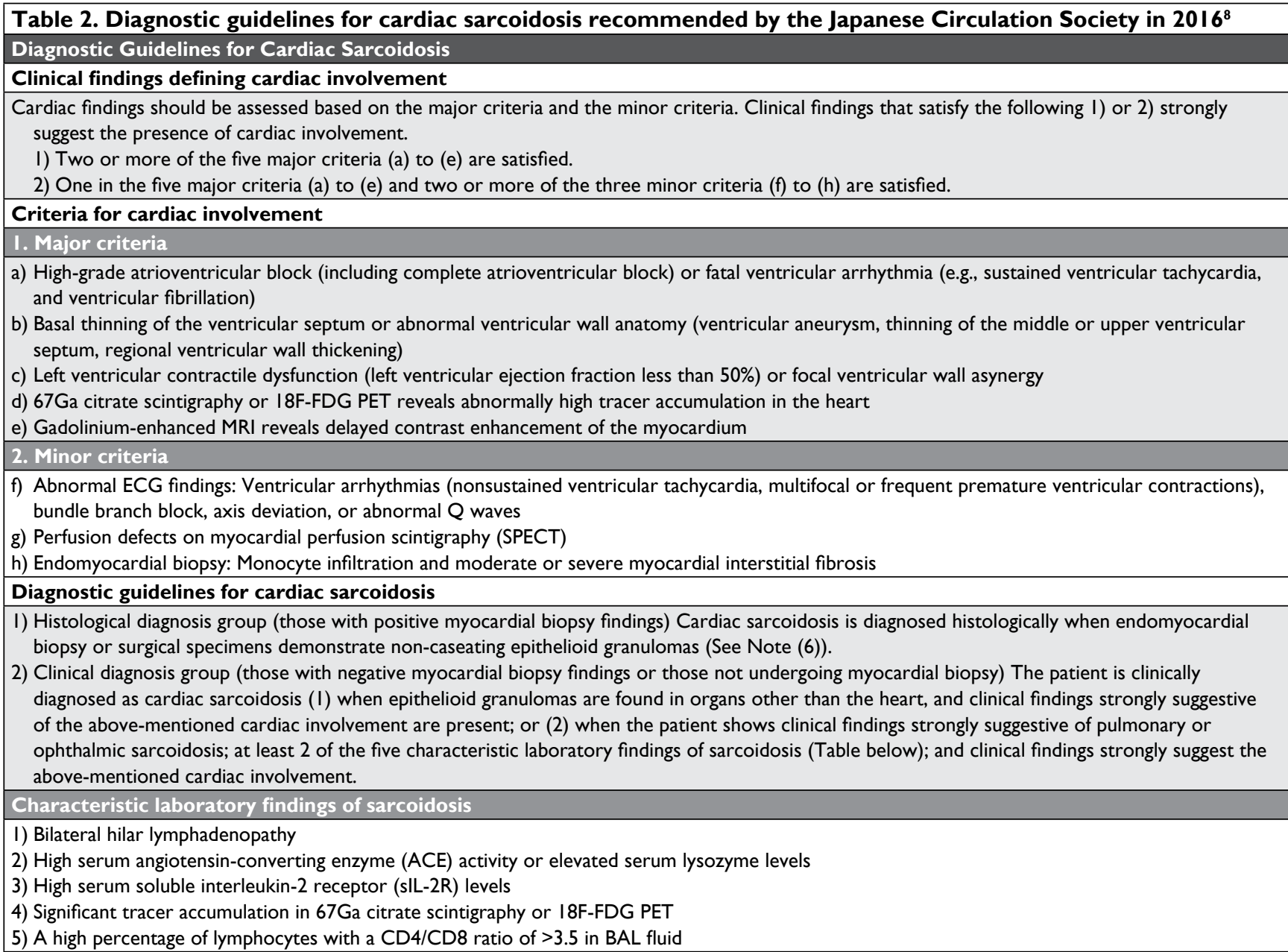

have been largely replaced by scar. However, different lesions and areas of lesions can be at different stages, and they evolve over time. Thus, the substrate for arrhythmia is very complex. It consists of areas with different degrees of active inflammation (acute, chronic, low-grade, high-grade), fibrosis and scar. Moreover, the arrhythmic substrate is dynamic: inflammation waxes and wanes, flares up. Importantly, myocardial reactivations frequently go undetected until arrhythmia or left ventricular systolic dysfunction become apparent. The location of lesions can be patchy, mesocardial, epicardial or endocardial. Lesions have a border zone that is typically irregular and heterogenous, and can also involve local Purkinje fibers. This substrate for ventricular arrhythmia is very different from the classical scar we see, for example, due to chronic myocardial infarction. With increased awareness, access to advanced imaging and earlier screening and diagnosis, it is likely that the patient population is changing from the cohorts reported $>10$ years ago - patients might present with more acute, actively inflamed substrate, instead of end-stage scar.

\section{CASE VIGNETTE \#I:}

34-year old athletic Caucasian male presents with new exertional dyspnea and lightheadedness. His ECG is shown in Figure 2. Note the markedly prolonged PR interval to $\sim 360 \mathrm{~ms}$ ( I ${ }^{\text {st }}$ degree atrioventricular (AV) block). Figure 3 shows rhythms strips obtained during exercise, showing a sudden drop in heart rate to approximately half. The strips show $2^{\text {nd }}$ degree type II AV block resulting in a dropped $7^{\text {th }} \mathrm{QRS}$, followed by 2:I AV block at peak exercise, associated with marked lightheadedness. Careful examination of the rate and the $T$ waves identifies blocked $P$ waves on top of the $T$ waves, which look slightly different when compared to $T$ waves during I-to-I AV conduction. These conduction abnormalities are, of course, markedly abnormal in a 34 year old and they will prompt further investigations. One of these investigations should be a cardiac 


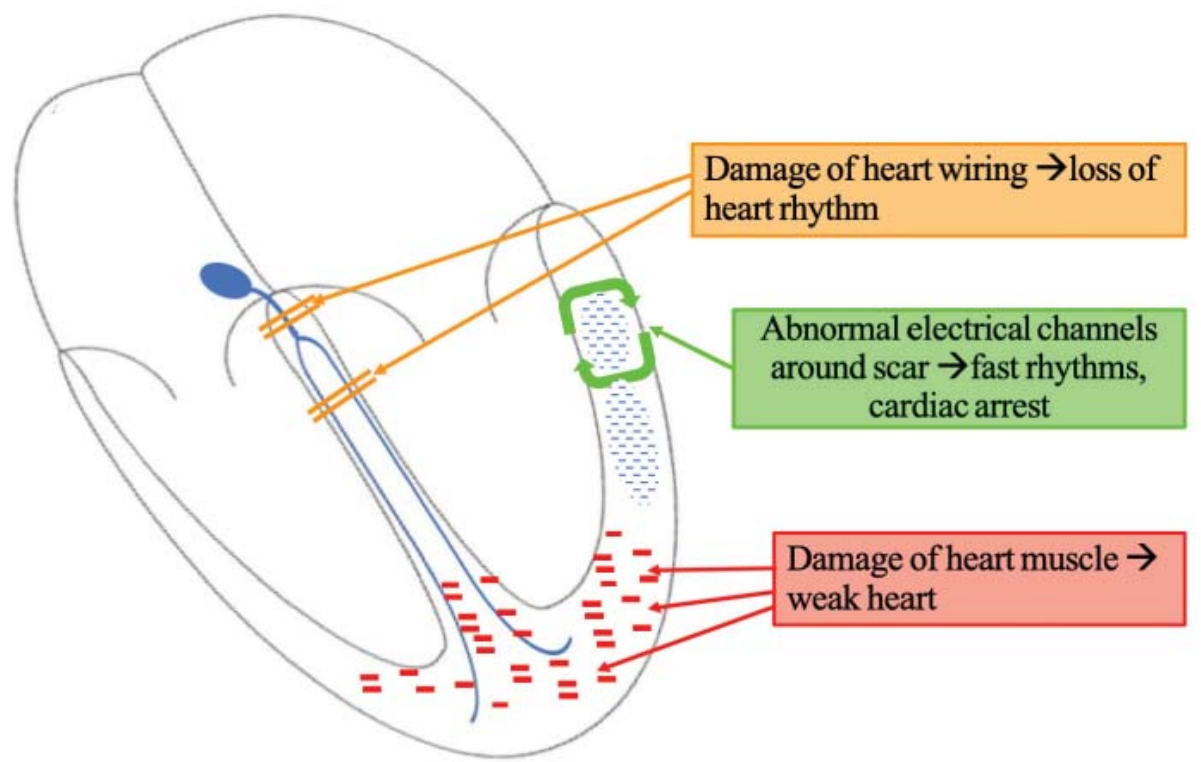

Figure I. Schematic representation used to explain potential cardiac complications to patients with systemic sarcoidosis.

MRI, as well as other tests looking for sarcoidosis. This patient had mediastinal lymphadenopathy and a small area of focal myocardial late Gadolinium enhancement (LGE) on cardiac MRI in the inferolateral wall (Figure 4). The MRI did not detect any lesions that explain the AV block. Involvement of the AV conduction system can be due to microscopic lesions that are below the detection threshold of our current imaging modalities. A transbronchial lung biopsy showed non-caseating granulomas and stains and cultures were negative for fungi and acid-fast bacilli (AFBs).

This case illustrates the need to investigate cardiac conduction abnormalities, especially AV block, if no obvious explanation is presenting itself. In a Finnish series of 133 patients aged 18 to 55 years who received pacemakers for second- or third-degree AV block, 72 patients had initially unexplained AV block. Of these, cardiac sarcoidosis was found in 19\%". In

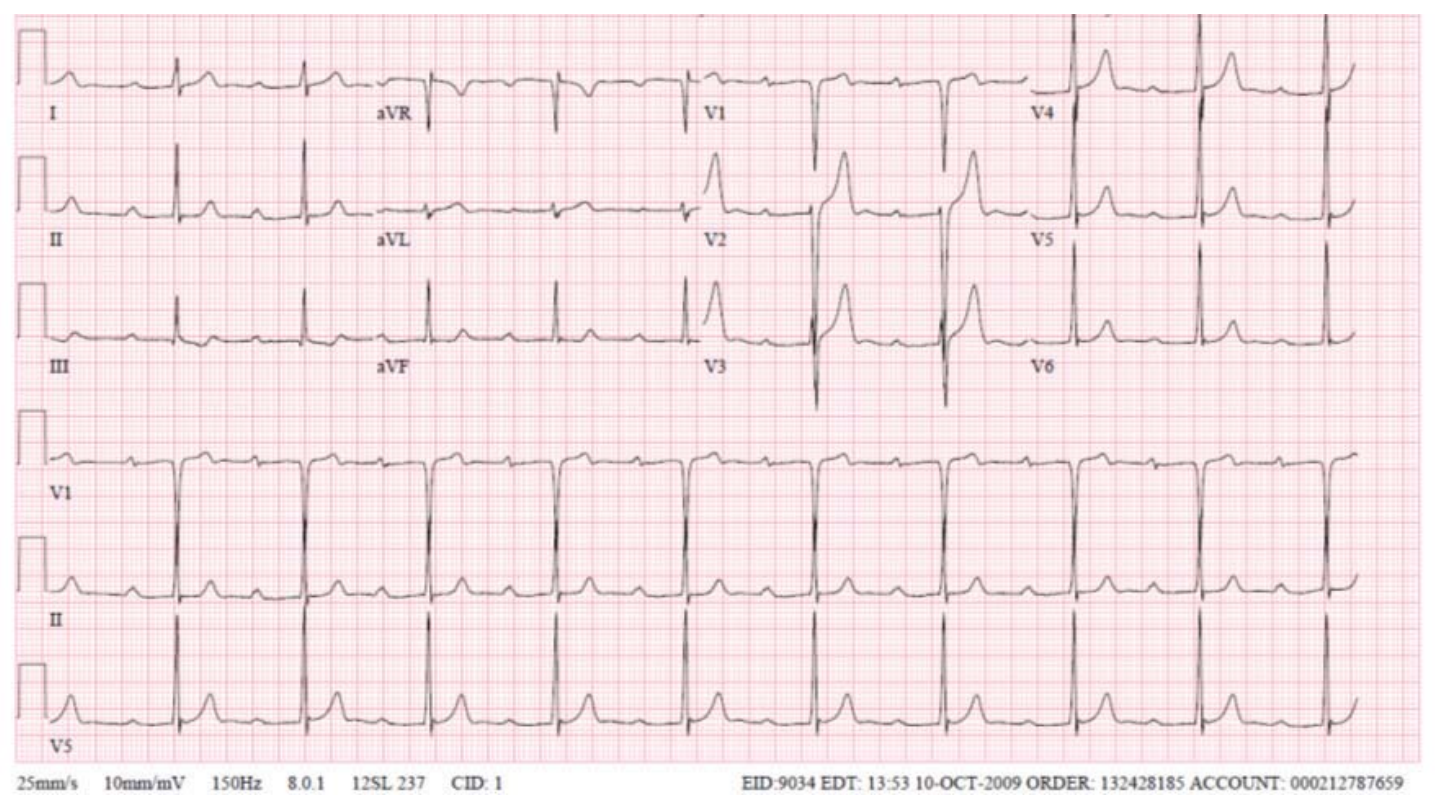

Figure 2. ECG: sinus rhythm with markedly prolonged PR interval to $\sim 360 \mathrm{~ms}$ (I st degree atrioventricular (AV) block). 
MIN R-R

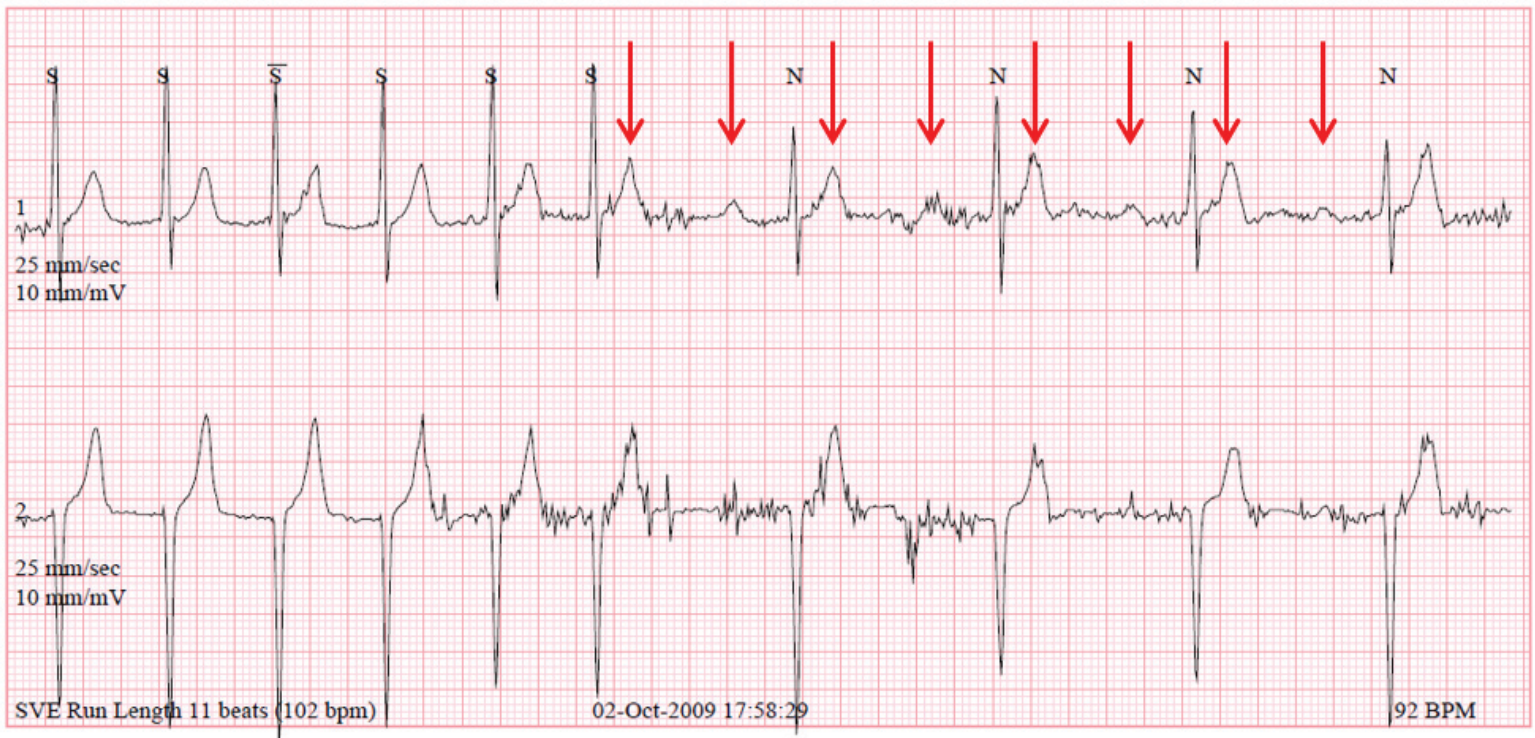

Figure 3. Rhythms strips obtained during exercise, showing $2^{\text {nd }}$ degree type II AV block resulting in a dropped 7th QRS, followed by 2:I AV block at peak exercise.

another series of 32 patients from Ontario, Canada aged 18-60 years who presented with unexplained $2^{\text {nd }}$ or $3^{\text {rd }}$ degree $\mathrm{AV}$ block and no previous history of sarcoidosis in any organ, $34 \%$ were diagnosed with cardiac sarcoidosis, all of which also had extra-cardiac involvement ${ }^{12}$.
Causes of AV block should be investigated in patients younger than 60 or 65 years old, if no obvious cause is present, such as aortic valve replacement, aortic valvular extensive calcification, acute myocarditis or endocarditis etc (see Table 3 ). We routinely obtain a cardiac MRI prior to device insertion if pos-
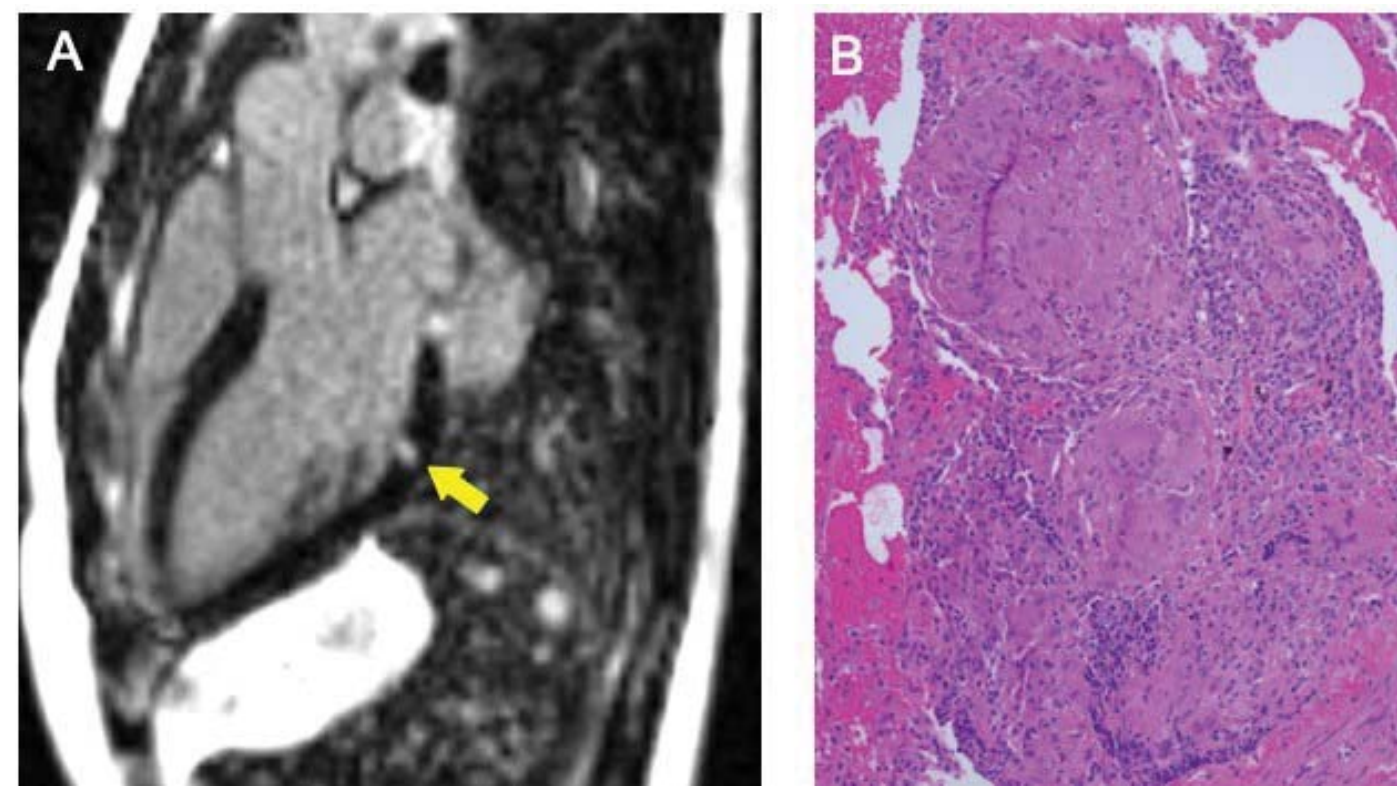

Figure 4. Panel A. Cardiac MRI for Case \#I. Note the focal area of abnormal delayed enhancement in the inferolateral wall towards the base. The stripe in the septum and blurring at the apex represent artifact. Panel B. Transbronchial lung biopsy - high-power section revealing noncaseating epithelioid granulomas (hematoxylin-eosin, $\times 200$ magnification). Special stains (Fite's acid fast, Grocott's Methenamine Silver and PAS Fungus) were negative for microorganisms. (courtesy of Jon W. Lomasney, MD). 


\section{Table 3: When should a cardiologist think of cardiac sarcoidosis?}

Screening in patients with cardiac presentation without known extracardiac sarcoidosis:

I. Patients $<60$ or 65 years (?) with unexplained high degree AV block (Mobitz II, $3^{\text {rd }}$ degree) ${ }^{11,12}$

2. Unexplained VT (if not typical benign idiopathic variety: outflow tract, fascicular or ischemic)*

3. Concomitant cardiomyopathy and heart block*

4. Concomitant ventricular arrhythmia and heart block*

5. Cardiomyopathy, ventricular arrhythmia or heart block in the presence of hilar or mediastinal lymphadenopathy (check chest imaging studies), uveitis, skin lesions, sinusitis etc. (screen for possible undiagnosed extracardiac sarcoidosis)

6. Unexplained cardiomyopathy, especially if myocardial infiltrates are seen on cardiac MRI (MRI or PET should be considered for any unexplained cardiomyopathy)

*other cardiomyopathies can be associated with conduction disease and ventricular arrhythmias: lamin mutation, myotonic dystrophy, ARVC, other genetic cardiomyopathies, amyloidosis, acute myocarditis, giant cell myocarditis etc.

sible, and if the MRI suggests cardiac sarcoidosis, we discuss with the patient the option of a pacemaker versus defibrillator. MRI can also be obtained after device insertion; we usually allow at least 2-3 months after device placement and use software to filter the artifact introduced by the metallic generator can. The devices are turned to asynchronous mode during MR imaging and parameters and programming are checked before and after MRI.

\section{CASE VIGNETTE \#2}

36 year old previously healthy Caucasian male presents with exertional palpitations. He is sent for a treadmill stress test. A 12-lead ECG obtained during exercise is shown (Figures 5 and 6). Note frequent PVCs with right bundle branch block-like morphology and superior axis suggesting a focus in the inferior left ventricle. The PVCs are relatively narrow and differential diagnosis at this point could include idiopathic, benign fascicu-

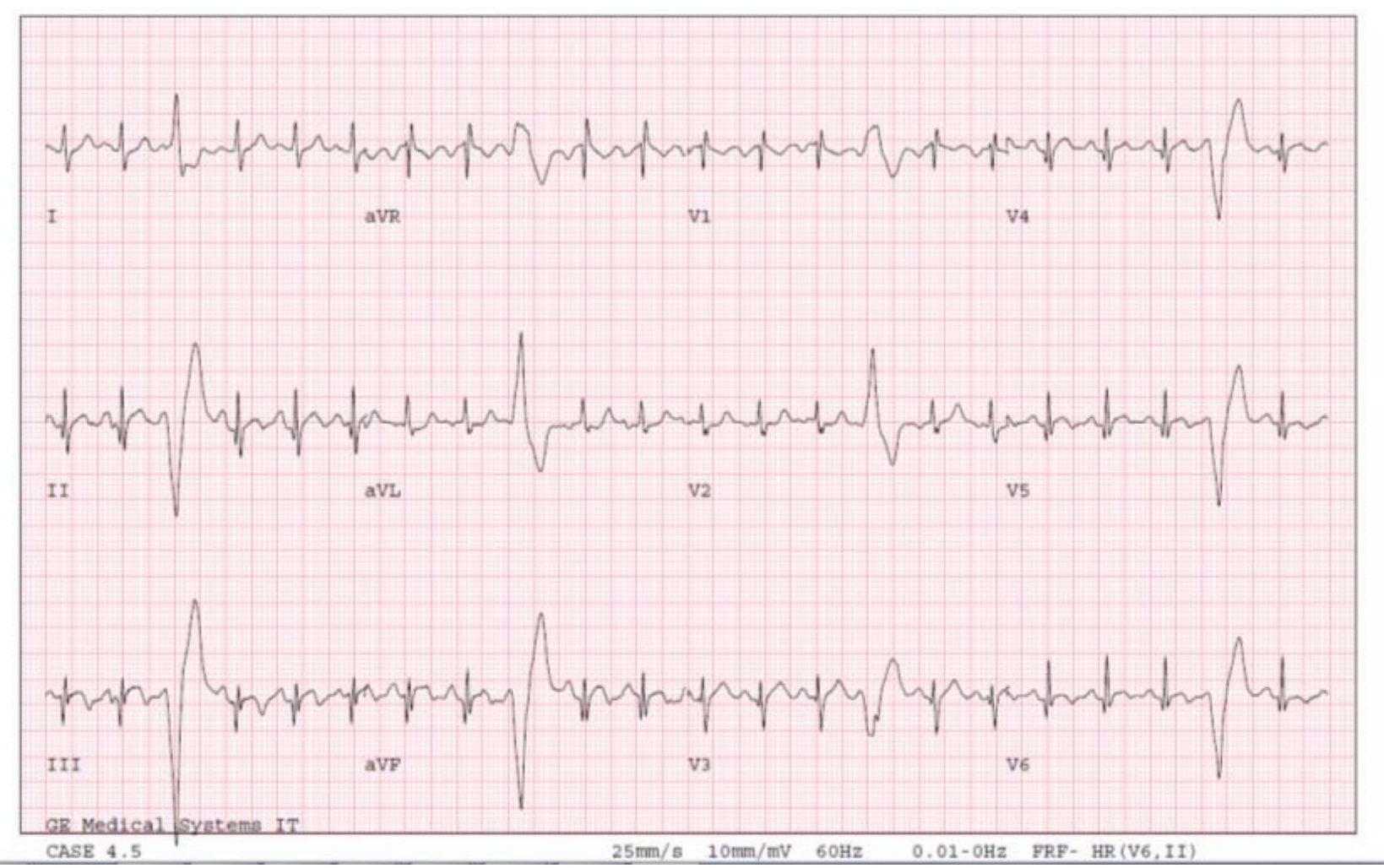

Figure 5. ECG obtained during exercise shows frequent PVCs with right bundle branch block-like morphology and superior axis and inferior $\mathrm{Q}$ waves. 


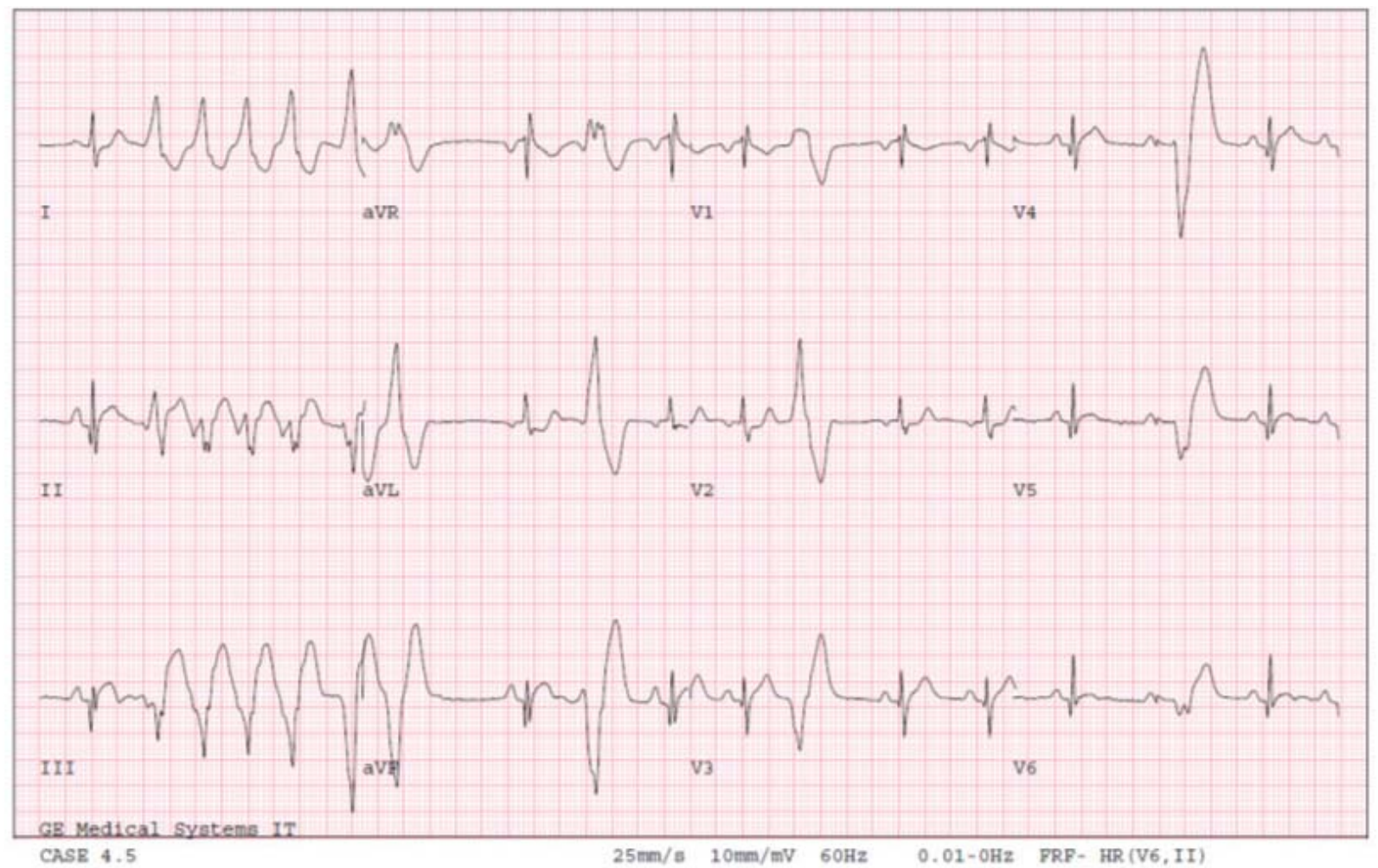

Figure 6. ECG during exercise shows paroxysmalVT with variable cycle length.
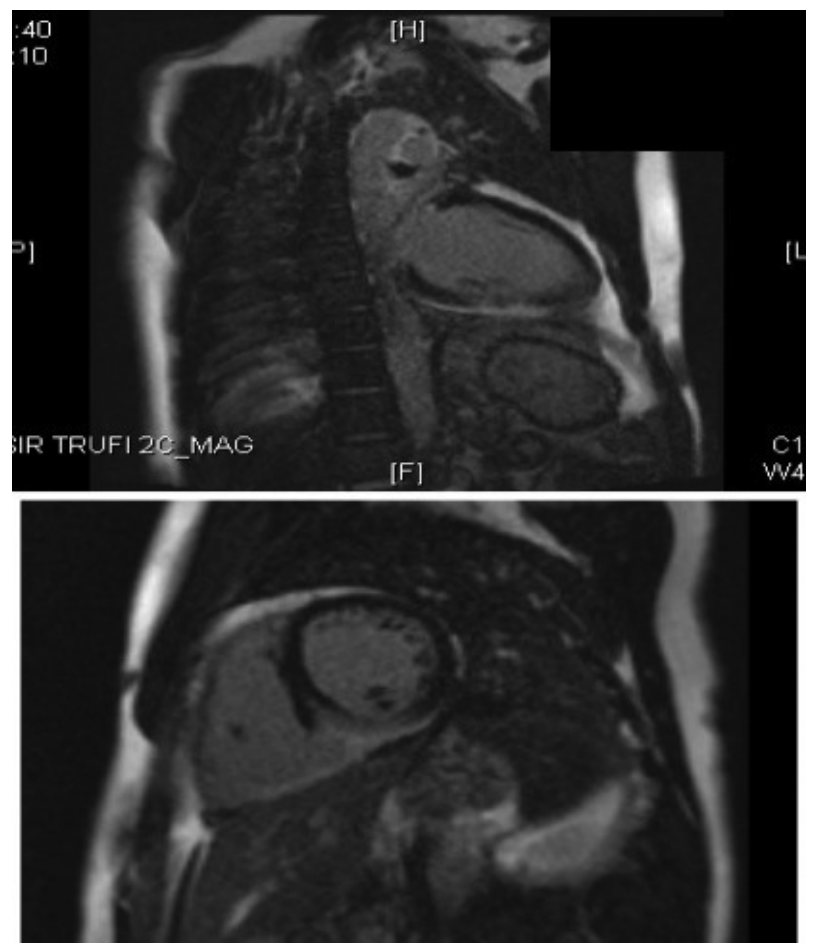

Figure 7. Cardiac MRI: mid-myocardial and epicardial delayed Gadolinium enhancement in infero-septum and LV inferior wall from mid-chamber to the apex, adjacent delayed enhancement of inferior wall of RV. LVEF was $61 \%$. lar PVCs. Note, however, the prominent inferior $\mathrm{Q}$ waves, with $Q$ amplitude greater than $1 / 3^{\text {rd }}$ of the $R$ wave. This suggests the presence of inferior wall scar. In Figure 6 we see a burst of paroxysmal VT with variable cycle length and somewhat pleomorphic QRS. This is suggestive of a focal tachycardia due to increased automaticity and is not a typical presentation of left posterior fascicular VT. The echocardiogram showed normal left ventricular ejection fraction (LVEF). A cardiac MRI, shown in Figure 7, also showed a normal LVEF of $61 \%$, but showed areas of mid-myocardial and epicardial delayed enhancement in infero-septum and LV inferior wall from mid-chamber to the apex, also with involvement of the adjacent inferior wall of the right ventricle. He had bilateral hilar lymphadenopathy and transbronchial biopsy was positive for non-caseating granulomas (stains and cultures were negative for fungi and AFBs).

\section{CASE VIGNETTE \#3:}

57 year old Caucasian female presents with syncope. Her presenting ECG is shown in Figure 8. Note the bifascicular block (right bundle branch block and left 
anterior fascicular block) and prolonged PR interval. In the hospital, transient complete heart block was noted on telemetry and a dual-chamber pacemaker was implanted. 18 months later, she complained of shortness of breath. Pacemaker interrogation showed that she was $100 \%$ paced in the right ventricle and her LVEF was $21 \%$. A coronary angiogram was normal. Her pacemaker was upgraded to a biventricular ICD. 6 months later, she received 2 appropriate ICD shocks for ventricular fibrillation and an Electrophysiology second opinion was obtained. Her LVEF was now $15 \%$. An FDG PET-CT was obtained, after preparatory diet to suppress myocardial glucose uptake and switch myocyte metabolism to fatty acids (protein and fat diet, no carbohydrates for 24-72 hours). This showed extensive foci of hypermetabolic lymphadenopathy in the mediastinum and hilar regions bilaterally, as well as extensive patchy uptake in both left and ri- ght ventricles. Transbronchial biopsy showed non-caseating granulomas (stains and cultures were negative for fungi and AFBs). She was aggressively treated with immunosuppressive therapy with PET-guided titration (see below) and, 8 more years later, her LVEF is $29 \%$ and has not had any more sustained VT/VF or ICD shocks.

This case presents a sequence of events that is quite common and illustrates the importance of screening for cardiac sarcoidosis in patients presenting with unexplained heart block (see Table 3). It also illustrates the role of anti-inflammatory, immunosuppressive therapy in the treatment of ventricular arrhythmias in the setting of active myocardial inflammation, and the increase in LVEF that can frequently be expected if therapy is instituted before permanent damage and scar are present.

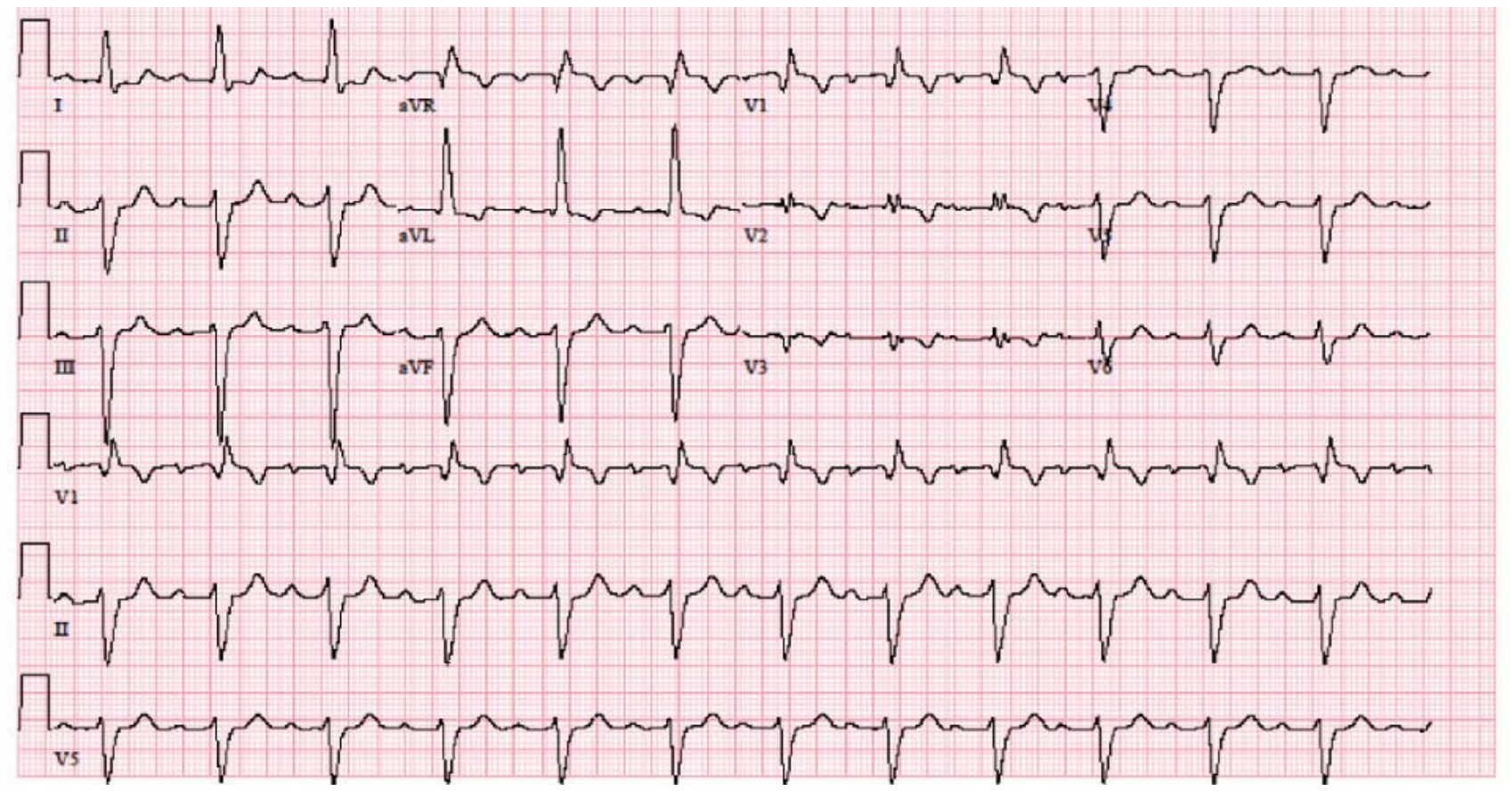

Figure 8. Sinus rhythm with bifascicular block (right bundle branch block and left anterior fascicular block) and prolonged PR interval (possible trifascicular block). 
Table 4. Screening in patients with any known extracardiac sarcoidosis

I. Symptoms: palpitations, syncope, dyspnea (unexplained by lung)

2. ECG:

- Frequency? $\rightarrow$ at least yearly.

- What abnormalities should prompt further evaluation?

I. AV block: even Ist degree, in the right clinical setting

2. Unexplained bundle branch block or new conduction disease, including fascicular block

3. PVCs that are not clearly benign (outflow tract or fascicular focus)

4. $Q$ waves

5. T wave inversions

\section{Holter monitor:}

- Obtain if palpitations/dizziness, consider routinely at initial diagnosis

- What abnormalities should prompt further evaluation?

I. VT

2. Pathologic $A \bigvee$ block (not physiologic: vagally-mediated, often nocturnal)

3. Frequent PVCs - unclear burden threshold, $>200 / 24$ hours (?)

4. Frequency? Unclear. Consider repeating if the patient has symptoms

\section{Echocardiogram:}

- Consider obtaining in all patients at initial diagnosis of extra-cardiac sarcoidosis

- Frequency: Initially and consider repeating if symptoms or in I-2 years (?)

- If any abnormality $\rightarrow$ Cardiology consultation, cardiac MRI and/or FDG PET-CT or PET-MR

\section{CASE VIGNETTE \#4}

45 year old Hispanic female who has been treated by Ophthalmology for stable sarcoid uveitis. She was asymptomatic, but a screening ECG was obtained according to practice guidelines discussed in the quarterly multidisciplinary, interdepartmental sarcoidosis conference held at the hospital. The ECG is shown in Figure 9. The ECG showed non-specific, but quite notable, $T$ wave inversions in the inferior and lateral leads and she was referred for further evaluation. Her echocardiogram showed normal LVEF $=55 \%$, but severe hypokinesis of the inferolateral LV wall was no-

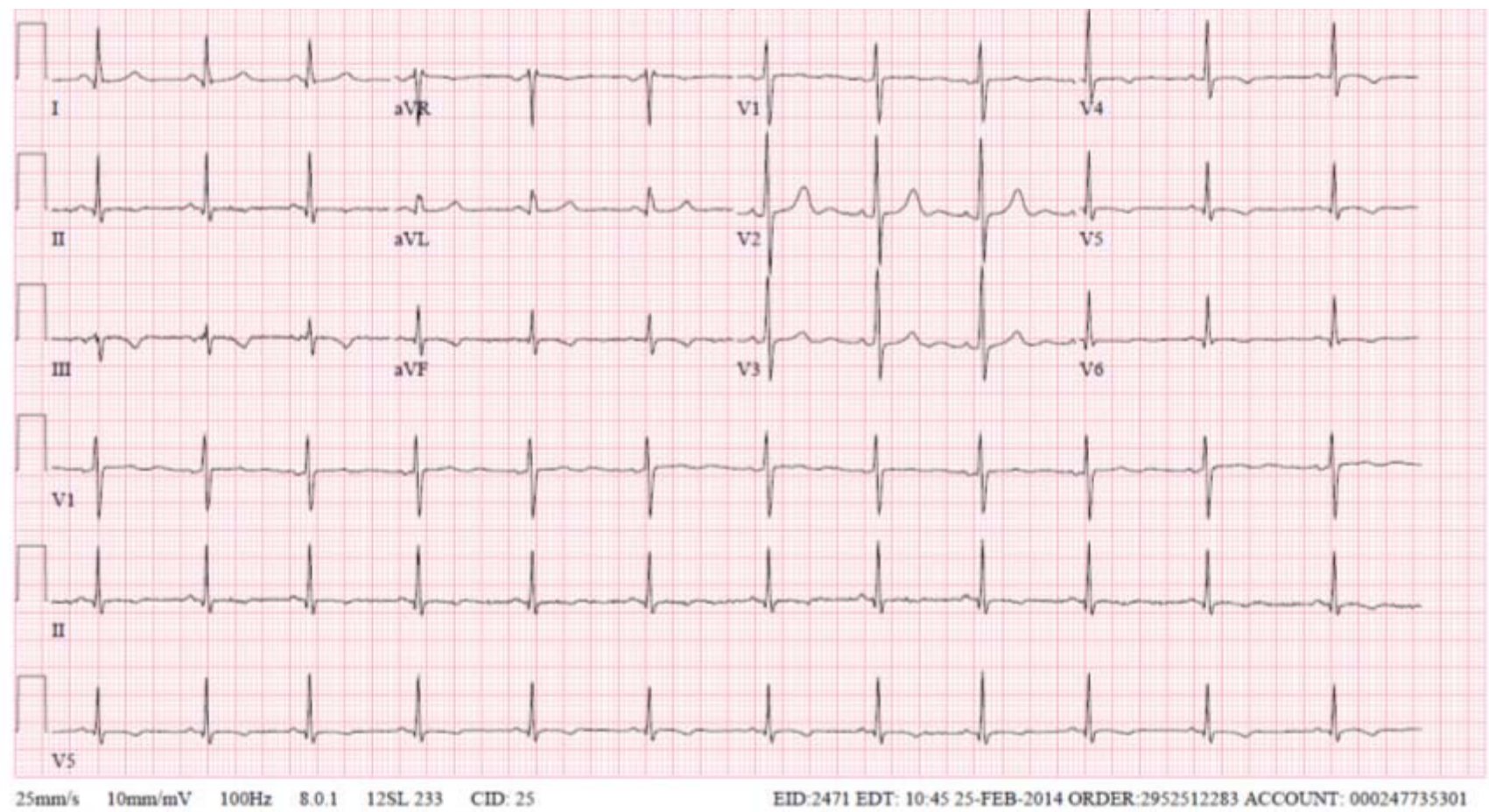

Figure 9. ECG showing T wave inversions in inferior and lateral leads. 


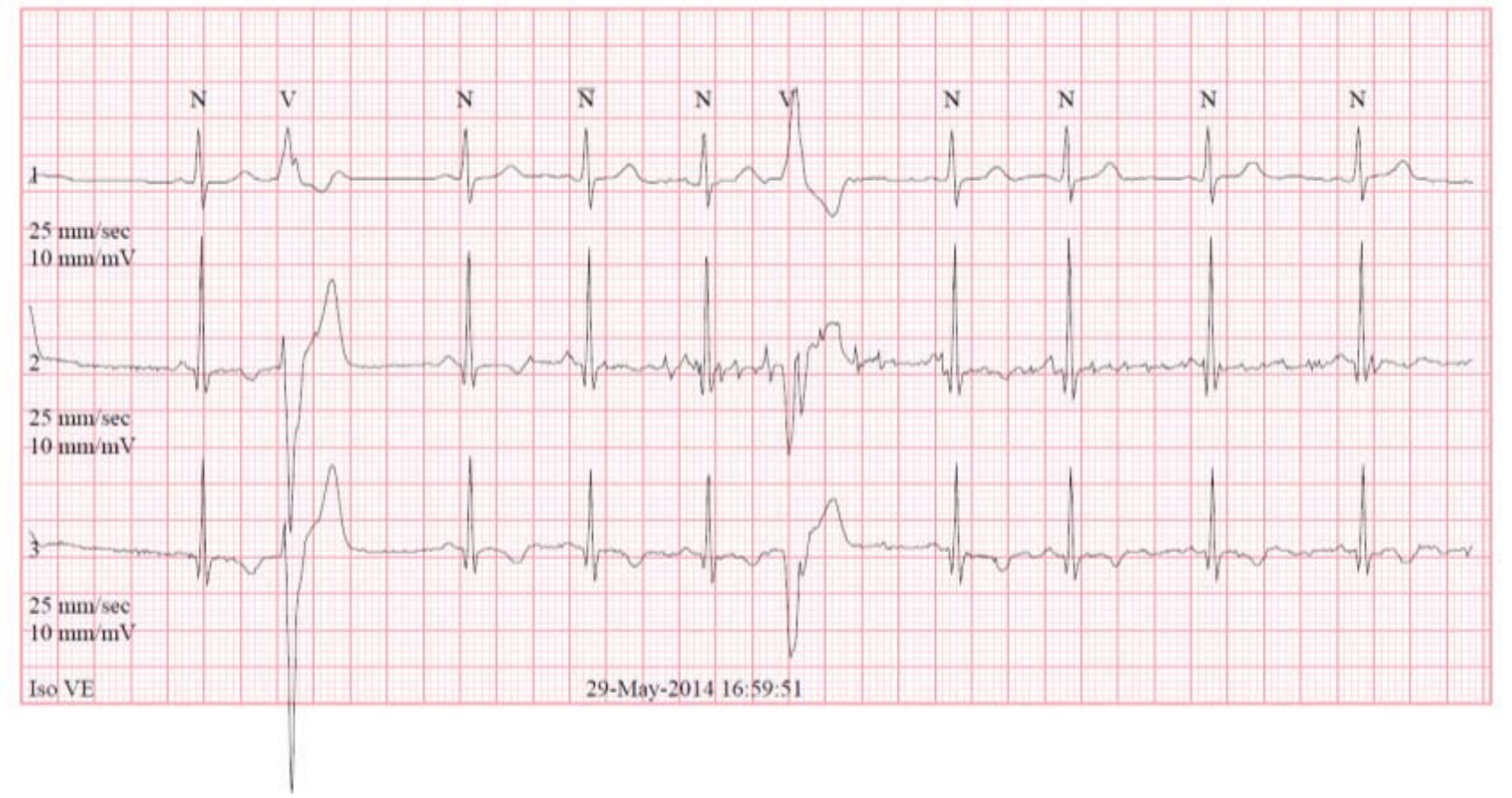

Figure 10. A 3-lead outpatient cardiac monitor also showed frequent PVCs, $\sim 5,000$ in 48 hours, with a superior axis QRS.

ted. A cardiac MRI was obtained, which showed LGE wall thinning and severe hypokinesis in the apical and mid-inferolateral LV wall. LVEF measured by MRI was 42\%. A 3-lead outpatient cardiac monitor also showed frequent PVCs, $\sim 5,000$ in 48 hours, with a superior axis QRS (Figure 10), compatible with a focus at the location of LGE on MRI. This case illustrates the importance of screening for cardiac involvement in patients with known sarcoidosis involving other organ systems (see Table 4).

\section{CASE VIGNETTE \#5:}

A 42 year old Caucasian male sees his doctor for screening because his mother has congestive heart failure and an idiopathic, non-ischemic cardiomyopathy. His LVEF is $40-45 \%$ by echocardiogram. A cardiac MRI showed multiple areas of patchy LGE (interventricular septum, anterolateral LV wall). An FDG-PET CT was also obtained. See Figure II: there was increased FDG uptake in the same areas noted on MRI. Familial or household clusters of sarcoidosis have been reported. However, this patient had no lymphadenophy and no extra-cardiac FDG uptake. A genetic test was obtained and it revealed a pathogenic desmoplakin mutation, consistent with a diagnosis of ARVC (arrhythmogenic right ventricular cardiomyopathy). The possibility

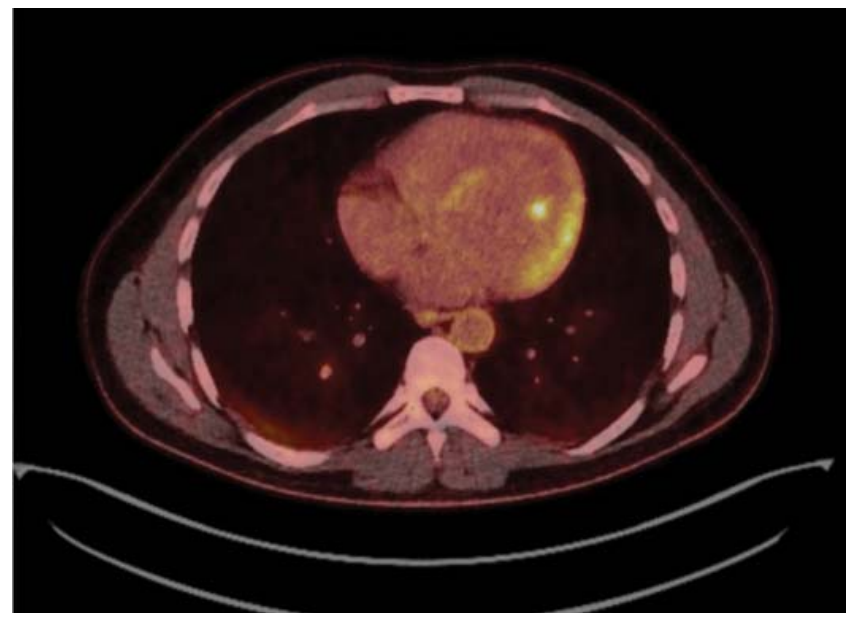

Figure I I. FDG-PET CT showed increased FDG uptake in the interventricular septum and anterolateral LV wall.

of positive FDG-PET in patients with ARVC has been reported by other investigators, as well ${ }^{13}$.

This case illustrates that not all positive myocardial FDG-PET scans represent cardiac sarcoidosis. Differential diagnosis includes some genetic cardiomyopathies, which may have areas of necrosis triggering local inflammation; other myocarditis etiologies, including viral, lymphocytic, giant cell myocarditis, etc. Importantly, recent radiofrequency ablation, in the preceding 3-6 months, can confound the picture by 
causing increased FDG uptake in the ablated areas. Careful clinical correlation should be made. Isolated cardiac sarcoidosis is relatively rare. In a international registry, 32 of 275 (12\%) patients with cardiac sarcoidosis had isolated cardiac involvement ${ }^{7}-$ and this is likely an over-estimate. This is one of the reasons why tissue diagnosis is important. It has been reported that bronchoaleolar lavage and transbronchial biopsy are frequently positive even if the PET scan does not show increased uptake.

\section{ROLE OF IMPLANTABLE DEFIBRILLATORS}

Once a diagnosis of cardiac sarcoidosis is made, it is important to assess the patient's risk of sudden death and whether an implantable cardioverter-defibrillator (ICD) is indicated (see Table 5).

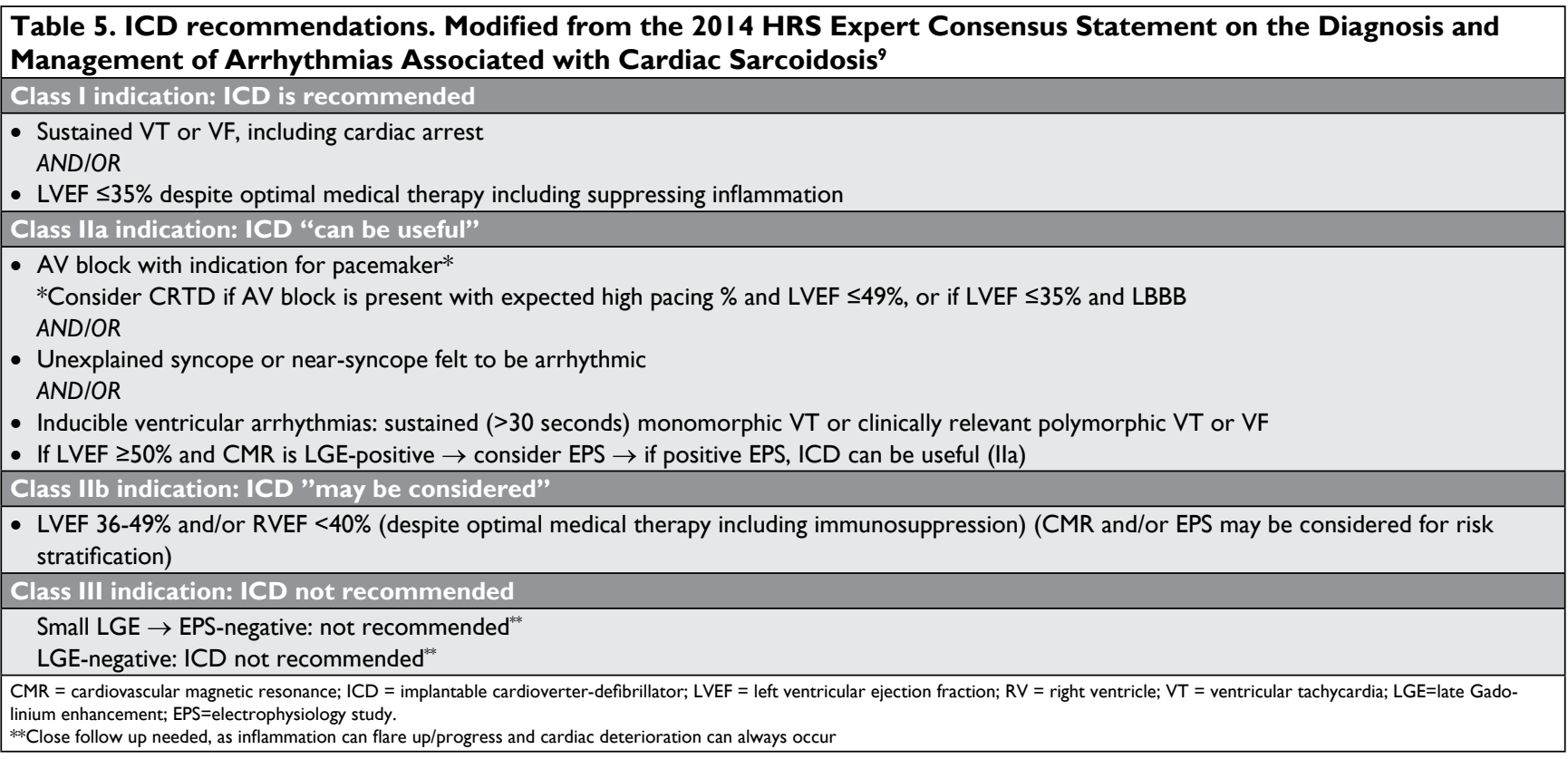

\section{ROLE OF ELECTROPHYSIOLOGY STUDY (EPS) IN RISK STRATIFICATION}

The role of programmed ventricular stimulation was investigated in a study ${ }^{14}$ of 76 patients with biopsyproven sarcoidosis who had evidence of cardiac sarcoidosis on PET or cardiac MRI. Patients with history of cardiac symptoms, prior ICD or ventricular arrhythmia were excluded. EPS for risk stratification was performed, consisting of the classic protocol with up to triple ventricular extrastimuli delivered from 2 different sites (right ventricular apical and outflow tract) at 2 different drive cycle lengths at baseline and on isoproterenol. Premature beats were delivered until refractoriness or to a minimum coupling interval of $200 \mathrm{~ms}$. If no sustained ventricular arrhythmias were induced, burst ventricular pacing was performed from cycle lengths of $300 \mathrm{~ms}$ to $220 \mathrm{~ms}$. If baseline stimulation failed to induce any arrhythmia, programmed stimulation was repeated during infusion with isoproterenol. The rate of infusion was titrated to increase sinus rate by $20 \% .8$ patients had inducible sustained VT or VF and received ICDs. The combined rate of ventricular arrhythmia or death was $75 \%$ in positive EPS group ( 2 deaths and 4 appropriate ICD shocks) and I.5\% in the negative EPS group. However, in this cohort, the group of patients with negative EPS had normal LVEF (56 $\pm 1.5 \%)$, while those with positive EPS had significantly decreased LVEF (36 $\pm 4.2 \%)$. Thus, it was not clear how much value the EP study provided in addition to a normal LVEF.

One other study' ${ }^{15}$ investigated the role of EPS in 69 patients with "probable" cardiac sarcoidosis in the absence of any AV block, VT/VF, LV dysfunction. Probable 
cardiac sarcoidosis was diagnosed based on biopsyproven extracardiac sarcoidosis in combination with abnormal PET or MR consistent with cardiac involvement ${ }^{9}$. In this cohort of patients with probable CS and preserved LV and RV function, a positive EPS was found to be predictive of sudden cardiac death or ventricular arrhythmias, while a negative EPS was associated with low incidence of these outcomes.

\section{CASE VIGNETTE \#6 (FOLLOW-UP OF THE SAME PATIENT DESCRIBED IN \\ CASE \#2)}

The patient underwent an EP study, which was technically negative for inducible sustained VT (only ,nonsustained" VT was induced, up to 19 seconds). He was then treated with prednisone, initially $60 \mathrm{mg}$ daily starting in January 2010 , followed by a slow taper until to October 2012. He was asymptomatic, competing in triathlons. He returns in September 2013 after a lapse in follow up. He described becoming tired perhaps more easily, but still training for triathlon. ICD interrogation showed that he had 10 seconds of VF with aborted ICD shock (Figure I2). It was also noted that the sensed $R$ wave amplitude had decreased (to 4-5 $\mathrm{mV}$, down from $12 \mathrm{mV}$ ). The sensed $R$ waves had been stable at $\sim 12 \mathrm{mV}$ until February 2013. A transthoracic echocardiogram was obtained in September 2013, which showed LVEF had now decreased to $15 \%$ (from 65\%). A myocardial FDG-PETCT in October 2013 showed extensive ventricular myocardial uptake (Figure 13).
Compare this to the patient's initial cardiac MRI from December 2009 (Figure 7), however, note that two imaging modalities show different things. Late Gadolinium enhancement (LGE) represents scar or late inflammation, while FDG uptake is a functional imaging modality and reflects metabolic activity of the inflammatory cells. We commonly see areas of LGE that are negative for FDG uptake and areas of positive FDG uptake without LGE.

This case illustrates that cardiac sarcoidosis is dynamic and unpredictable, and close surveillance for response to therapy and monitoring for reactivation is necessary.

This patient went on to have multiple ICD shocks for VF and VT despite aggressive medical therapy with high doses prednisone $(40-60 \mathrm{mg} /$ day) and methotrexate, in addition to amiodarone 200-400 $\mathrm{mg} /$ day and mexiletine $150 \mathrm{mg}$ TID. His arrhythmias did subside eventually and LVEF improved to $25 \%$. It has been reported that cardiac sarcoidosis is highly arrhythmogenic, more so than fixed scar (such as healed myocardial infarction), likely due to the multifocal, inflammatory, evolving nature of the proarrhythmic substrate. In a cohort ${ }^{16}$ of 235 patients followed for $4.2 \pm 4.0$ years, $36 \%$ received appropriate ICD therapy. One should have a relatively lower threshold for recommending ICD in these patients. Many or most patients will probably benefit from an ICD, including those with normal LVEF but who have significant LGE on MRI or positive EP study (see Table 5). It may be easier to identify those who most likely will not benefit from ICD - see Table 6.

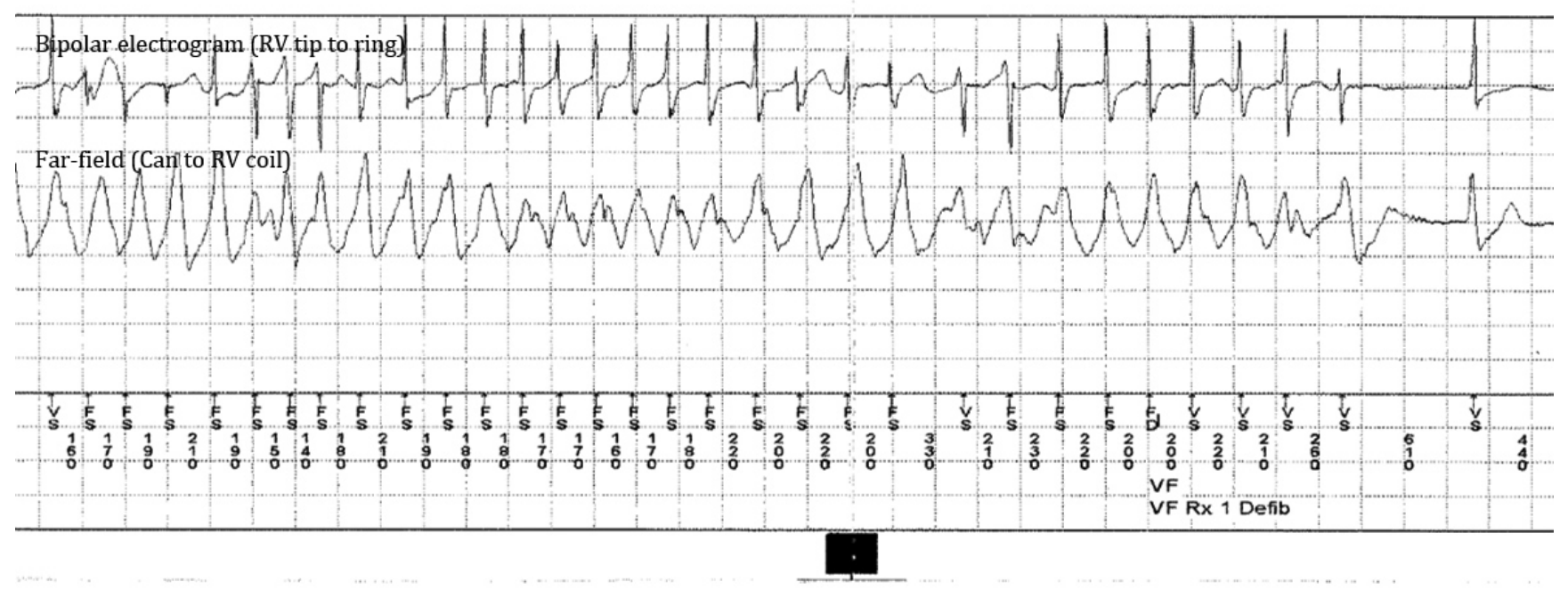

Figure I2. ICD interrogation shows non-sustained VF with aborted ICD shock. 
Table 6. An ICD is not indicated if:

All of these are true:

- No syncope

- Normal LVEF/RVEF

- No or minimal LGE on MRI or FDG uptake on PET

- Negative EP study

- No indication for pacing

- Patient will be closely monitored for disease progression

\section{FDG-PET (October 2013)}
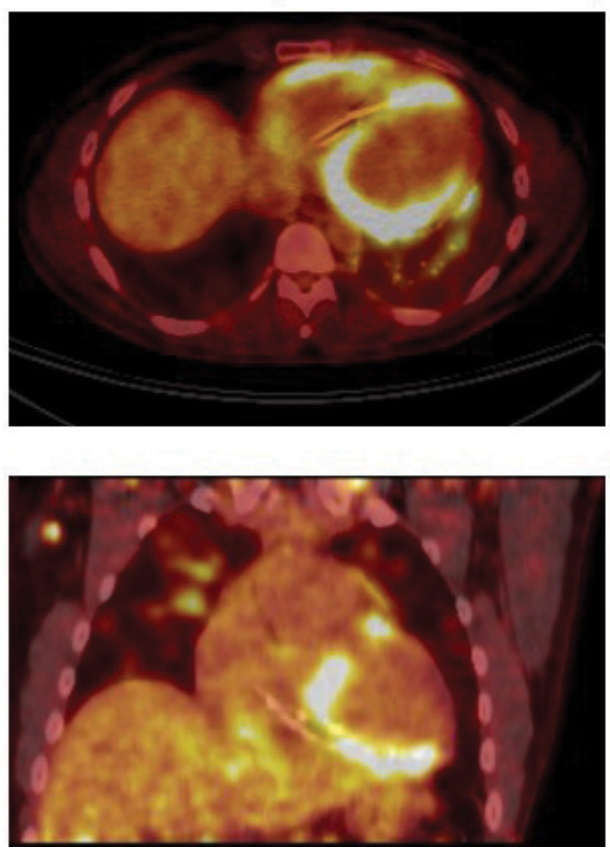

Figure 13. FDG-PET from October 2013: Note the extensive and prominent FDG uptake in both LV and RV, sparing some of the anterolateral wall of the LV.

\section{TREATMENT OF VENTRICULAR ARRHYTHMIAS IN CARDIAC SARCOIDOSIS}

In addition to ICD, we should always consider the following tools that are available to treat VT in these patients. Even if an ICD is inserted, it obviously does not actually solve the VT problem, and if VT is left untreated, the patient is exposed to the potential of multiple ICD shocks.

I. Immunosuppression (corticosteroids, methotrexate, mycophenolate, Infliximab ${ }^{17}$ )

2. Antiarrhythmic drugs: sotalol, amiodarone, (mexiletine)

\section{Ablation}

Remarkably, there are no randomized, controlled clinical trials of immunosuppression. Different centers use different protocols and staged approaches. Corti- costeroid are the mainstay of therapy. Steroid-sparing agents are frequently added as the steroids are tapered down. More detailed discussions of immunosuppression for cardiac sarcoidosis can be found elsewhere ${ }^{18}$. Long-term immunosuppressive therapy is a complex treatment requiring specialized skill and should be directed by physicians with expertise and experience, usually rheumatologists, pulmonologists with experience managing pulmonary sarcoidosis, or cardiologists with experience managing heart transplant patients. I will mention here the potential role of FDG-PET guidance in titrating and adjusting immunosuppressive therapy. This is done in the idea that suppression of FDG uptake correlates with suppression of inflammation, which might prevent progressive myocardial damage and facilitate healing of reversible injury ${ }^{19}$.

Figure 14 shows serial FDG-PETCT images obtained in one patient. He was initially treated with prednisone $60 \mathrm{mg}$ daily (some reports suggest that initial prednisone dose of $30 \mathrm{mg}$ daily might be equally good).

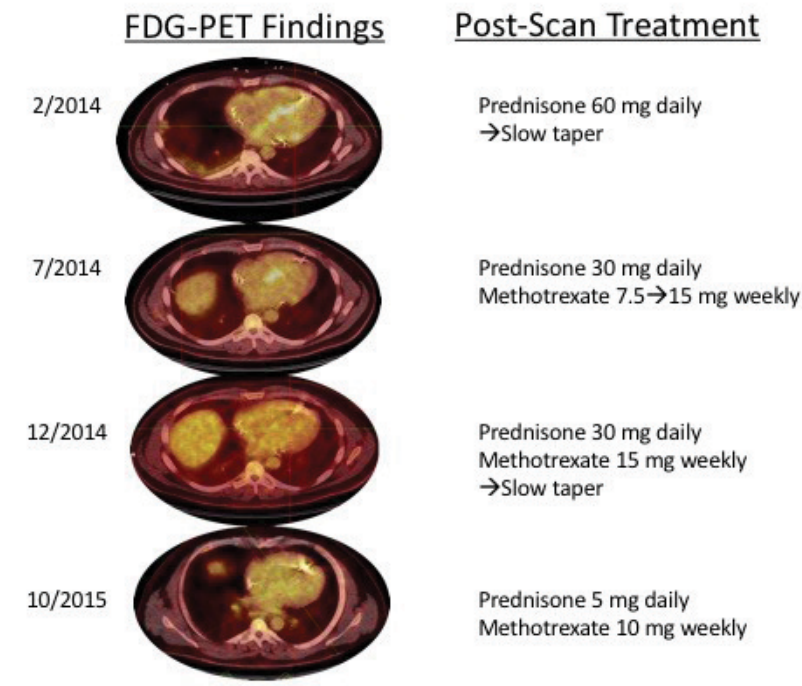

Figure 14. Cardiac outcomes using FDG-PET-guided immunosuppression. FDG-PET scan treatment series in one representative patient. Initial scan demonstrates myocardial inflammation at the base, localized predominantly to the anterior, septal and inferior walls of the left ventricle. Complete suppression is achieved on the third scan and maintained on the 4th scan. The post-scan immunosuppressive regimen, sequence in treatment series, and date are listed in association with each scan. 


\begin{tabular}{|c|c|c|c|}
\hline Adverse outcomes depe & whether & f FDG-PET myocardial activity wa & as achiev \\
\hline & & $\begin{array}{l}\text { Complete PET suppression not } \\
\text { achieved }\end{array}$ & $P$ value \\
\hline Death or Heart Transplant & I (0.03\%) & $4(27 \%)$ & $<0.05$ \\
\hline CAO & $9(33 \%)$ & $10(67 \%)$ & $<0.05$ \\
\hline Sustained VT/VF & $6(22 \%)$ & $8(53 \%)$ & $<0.05$ \\
\hline
\end{tabular}

The initial scan demonstrates myocardial inflammation at the base of LV, localized predominantly to the anterior, septal and inferior walls of the left ventricle. Complete suppression is achieved on the third scan and maintained on the $4^{\text {th }}$ scan. The post-scan immunosuppressive regimen, sequence in treatment series, and date are listed in association with each scan.

In a series of 42 patients $^{20}$, we found that using FDGPET scans to monitor myocardial inflammation and to titrate immunosuppressive therapy may be beneficial in patients with cardiac sarcoidosis (results presented in Table 7). Complete suppression of myocardial inflammation was associated with low risk of adverse outcomes and death during follow up. Conversely, failure to obtain complete suppression of myocardial inflammation was associated with increased risk of adverse outcomes and death. Surveillance FDG-PET scanning can identify patients who might have steroid-resistant form of disease and require additional or alternative agents. In conclusion, immunosuppression plays an important antiarrhythmic role in cardiac sarcoidosis when active myocardial inflammation is present. Arrhythmia-free course seems to correlates with the ability to suppress inflammation.

The value of FDG-PET-guided immunosuppression has to be balanced against its high cost and significant radiation dose if repeated scans are obtained (the dose can be reduced at follow-up scan by obtaining limited cardiac scans, rather than whole-body). The optimal interval between scans is unknown; one possible strategy would employ repeat at 4-6 months initially, then only if clinical, echocardiographic or other deterioration is noted. Most importantly, there is a great need for prospective clinical trials, since all the available data consists of retrospective studies. The role of FDG-PET in cardiac sarcoidosis has been detailed in a 2017 expert consensus document ${ }^{2 !}$.

\section{ROLE OF ABLATION FOR VENTRICULAR ARRHYTHMIAS IN CARDIAC SARCOIDOSIS}

Ablation is not a default first line therapy in many or most patients with cardiac sarcoidosis. The overall strategy has to be tailored according to the clinical scenario and underlying mechanisms (see Table 8).

Most series of VT ablation published in the literature are small. Patients who underwent ablation presented with incessant VT or VT storm (10-30\%), most frequently the arrhythmias were scar-related - and multiple morphologies were inducible. Other noted VT mechanisms were Purkinje fiber-related, and included bundle branch reentry and microreentry, as well as non-reentrant Purkinje foci.

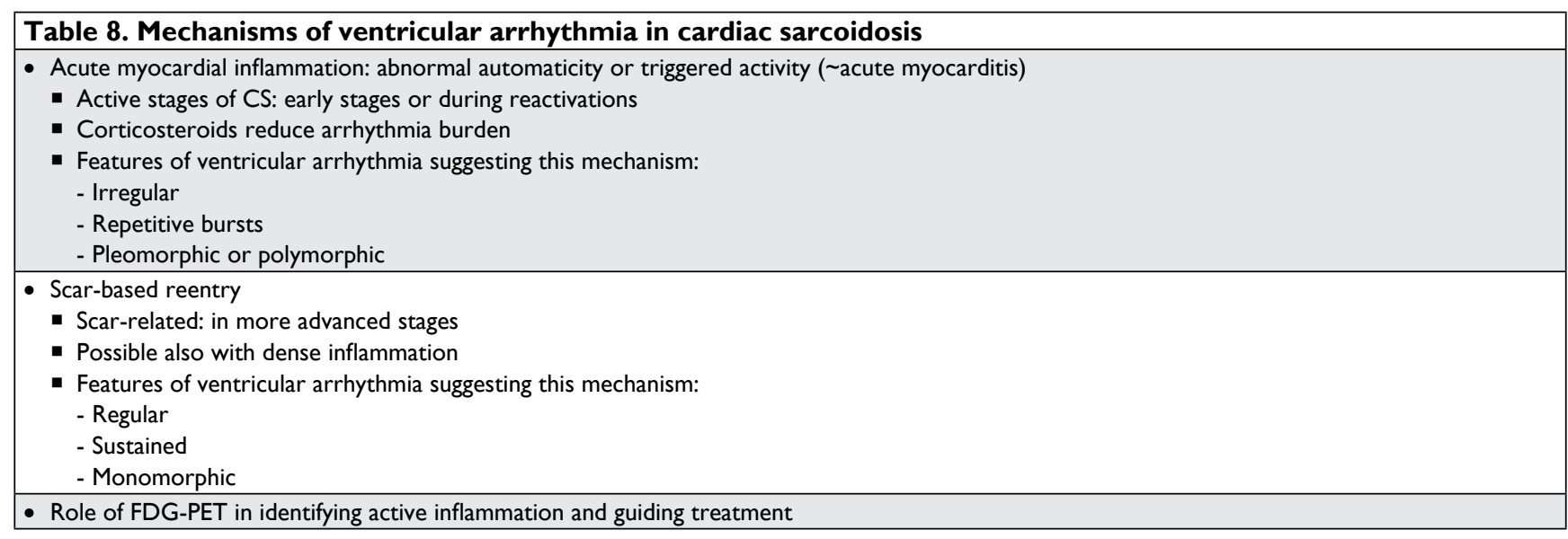


- During median 2.5-year follow-up, $8 \mathrm{I}$ (5I\%) patients experienced VT recurrence, heart transplantation or death

- LV dysfunction and inflammation in pre-procedural I8F-FDG PET were significantly associated with adverse prognosis

- Appropriately timed ablation procedures remain important in the management of CS-related VT in conjunction with medical therapy.

We investigated the role of VT ablation in a multicenter study from international Cardiac Sarcoidosis Consortium ${ }^{22}$. This is a multicenter study of 158 patients (age $52 \pm$ II years, $33 \%$ female) who underwent VT ablation. The median time from cardiac sarcoidosis diagnosis to VT was 827 days (interquartile range 210-I,676). There was a high rate of VT recurrence, especially in patients with FDG-PET evidence of active inflammation. In this multicenter registry reflecting real-world practice internationally, catheter ablation treatment of cardiac sarcoidosis -related VT remained challenging and rates of recurrence and repeat procedures were high. Survival curves are shown in Figure 15 and conclusions are summarized in Table 9.

To summarize, the management of ventricular arrhythmias in patients with cardiac sarcoidosis should follow a stepwise approach tailored to the individual patient.

First, one has to assess the risk of sudden death, even in asymptomatic patients, and consider the po-
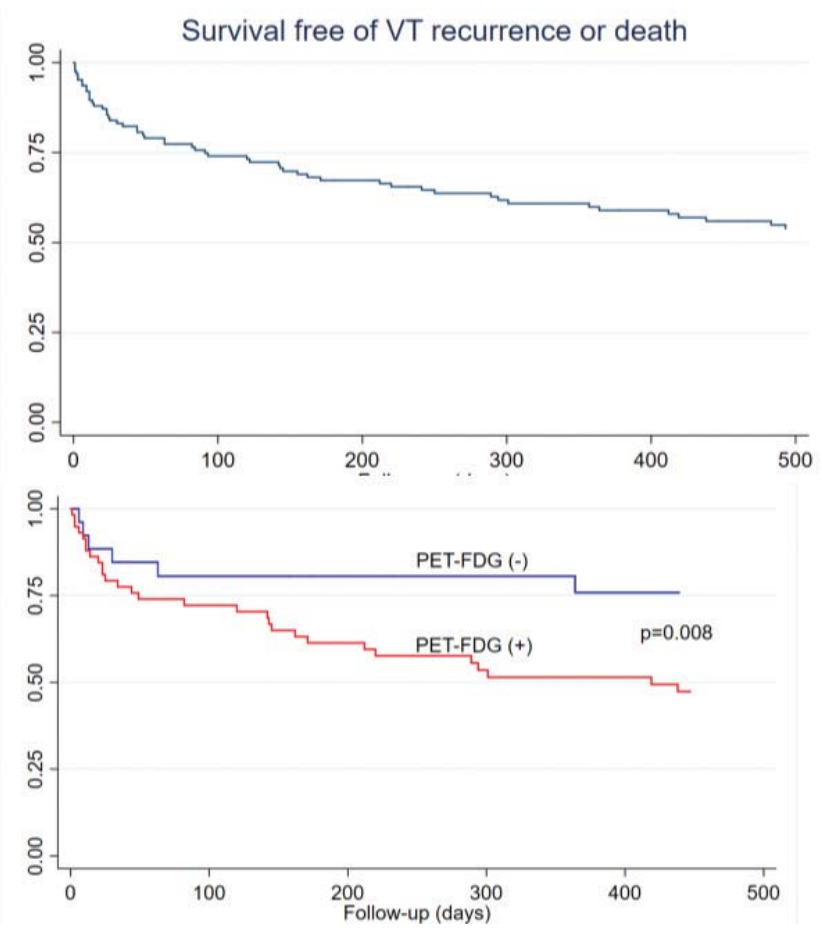

Figure 15. Event-free survival curves afterVT ablation. Data from the Cardiac Sarcoidosis Consortium ${ }^{22}$. (Reproduced with permission) tential benefit for ICD. The ICD may be life-saving, but it does eliminate the arrhythmia, and these patients have a high rate of ICD shocks, so more needs to be done if arrhythmias are present. Antiarrhythmic drug therapy is important. Amiodarone is the mainstay of antiarrhythmic therapy, especially acutely, or in patients with decreased LVEF, and in most patients with active inflammation while allowing time for immunosuppressive therapy to work. The patient should be monitored closely for liver toxicity and other side effects of amiodarone. This can be later switched to sotalol - particularly in younger patients and if the arrhythmias subside, in order to avoid the toxicity of long-term amiodarone. In all patients with cardiac sarcoidosis presenting with arrhythmia, it is important to assess for active myocardial inflammation. If it is present, first line of therapy should include immunosuppression and antiarrhythmic drug therapy. Ablation can be considered if necessary, in situation such as VT storm unresponsive to medical therapy or recurrent VF with an identifiable PVC trigger (see Table 10). If no active myocardial inflammation is present, antiarrhythmic drug therapy and ablation are useful tools, in addition to careful programming of the ICD (maximizing anti-tachycardia pacing, increasing lower rate limits to preempt post-PVC pauses, etc.)

\section{CONCLUSION}

Cardiac sarcoidosis is an underdiagnosed disease with protean manifestations. The prevalence of cardiac sarcoidosis in Romania is not known, but a significant number of patients might be currently under the care of cardiologists, cardiac electrophysiologists and other medical specialists. Identifying and treating these patients could impact significantly their clinical course, morbidity and mortality. Once a diagnosis is made, cardiac sarcoidosis has a dynamic and unpredictable course. Diagnosis and treatment requires a multidisciplinary team of medical specialists in cardiology, cardiac electrophysiology, pulmonology, rheumatology and others. Arrhythmias in cardiac sarcoidosis have a variety of mechanisms and are associated with poor prognosis and significant risk of death. Arrhyth- 


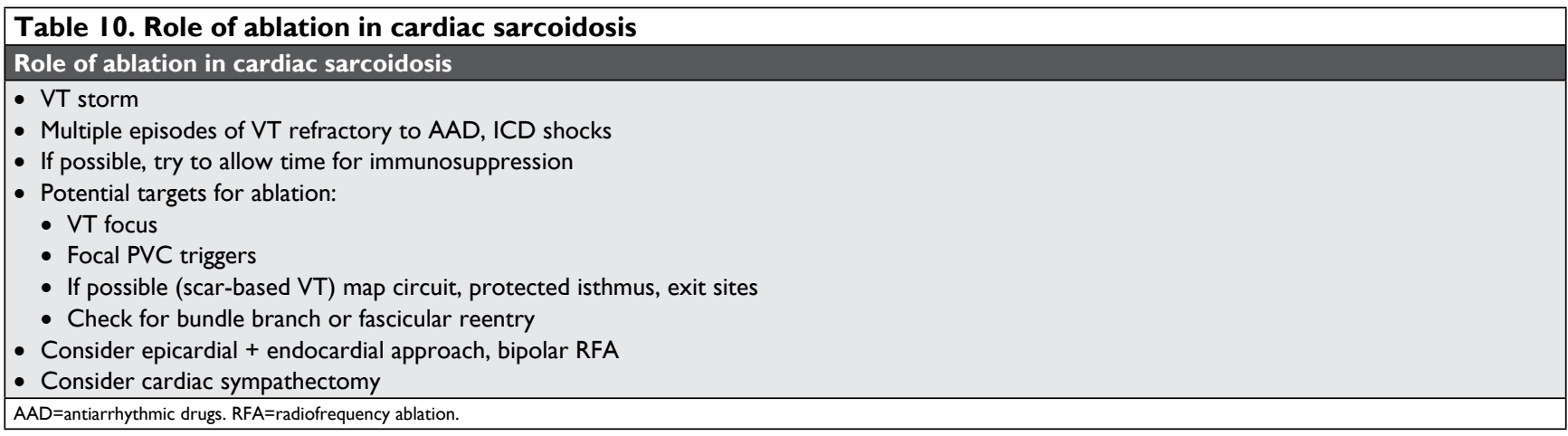

mia management tools include ICDs, immunosuppression, antiarrhythmic drugs and ablation. These should be used in an individualized fashion, according to the clinical presentation and the complex underlying pathophysiology.

Conflict of interest: none declared.

Acknowledgement: The author would like to thank Prof. Tiberiu Nanea, Prof. Pompiliu Popescu, Prof. Carmen Fierbinteanu Braticevici and others who inspired him during medical school.

\section{References}

I. Strambu I, Salmen T, Traila D and Croitoru A. Romanian Registry for Interstitial Lung Diseases (REGIS): inclusion of patients in 3 years [Abstract]. Eur Respir ]. 2017;50:PA868.

2. Salmen T, Traila D and Strambu IR. The Results of a Three Year Analysis on Sarcoidosis Patients Registered in the Regis Electronic Registry. Medicina Interna. 2018;15:7-13.

3. Hillerdal G, Nou E, Osterman K and Schmekel B. Sarcoidosis: epidemiology and prognosis. A 15 -year European study. Am Rev Respir Dis. 1984;130:29-32.

4. Deubelbeiss U, Gemperli A, Schindler C, Baty F and Brutsche MH. Prevalence of sarcoidosis in Switzerland is associated with environmental factors. Eur Respir J. 2010;35:1088-97.

5. Beghe D, Dall'Asta L, Garavelli C, Pastorelli AA, Muscarella M, Saccani G, Aiello M, Crisafulli E, Corradi M, Stacchini P, Chetta A and Bertorelli G. Sarcoidosis in an Italian province. Prevalence and environmental risk factors. PLoS One. 2017;12:e0176859.

6. Erdal BS, Clymer BD, Yildiz VO, Julian MW and Crouser ED. Unexpectedly high prevalence of sarcoidosis in a representative U.S. Metropolitan population. Respir Med. 2012;106:893-9.

7. Crawford TC FW, Kron J, Chicos AB, Ellenbogen KA, Kalbfleisch SJ, Vedantham V, Roukoz H, Benditt DG, Estes NAM, Sauer WH, Soejima K, Bhan A, Murgatroyd F, Ortman M, Rosenfeld LE, DeLurgio BD, MD, Kaitani K, Zimetbaum PJ, Nour K, Goldberger ZD, Patton KK, Dickfeld TM, Mazzini MJ, Frisch DR, Mukerji SS, Narasimhan C, Steckman D, Marcotte K, Gu X, Bogun FM. Patients with Cardiac Sarcoidosis Experience a Significant Delay in Diagnosis. Heart Rhythm. 2017;14:S8I.

8. Terasaki F, Azuma A, Anzai T, Ishizaka N, Ishida $Y$, Isobe M, Inomata T, Ishibashi-Ueda H, Eishi Y, Kitakaze M, Kusano K, Sakata Y, Shijubo N, Tsuchida A, Tsutsui H, Nakajima T, Nakatani S, Horii T, Yazaki Y, Yamaguchi E, Yamaguchi T, Ide T, Okamura H, Kato Y, Goya M, Sakakibara M, Soejima K, Nagai T, Nakamura H, Noda T, Hasegawa T, Morita H, Ohe T, Kihara Y, Saito Y, Sugiyama Y, Morimoto SI, Yamashina $A$ and Japanese Circulation Society Joint Working G. JCS 2016 Guideline on Diagnosis and Treatment of Cardiac SarcoidosisDigest Version. Circ J. 2019;83:2329-2388.
9. Birnie DH, Sauer WH, Bogun F, Cooper JM, Culver DA, Duvernoy CS, Judson MA, Kron J, Mehta D, Cosedis Nielsen J, Patel AR, Ohe $T$, Raatikainen $P$ and Soejima K. HRS expert consensus statement on the diagnosis and management of arrhythmias associated with cardiac sarcoidosis. Heart Rhythm. 2014;1 I:1305-23.

10. Judson MA, Costabel U, Drent M, Wells A, Maier L, Koth L, Shigemitsu $H$, Culver DA, Gelfand J, Valeyre D, Sweiss N, Crouser E, Morgenthau AS, Lower EE, Azuma A, Ishihara M, Morimoto S, Tetsuo Yamaguchi T, Shijubo N, Grutters JC, Rosenbach M, Li HP, Rottoli P, Inoue Y, Prasse A, Baughman RP and Organ Assessment Instrument Investigators TW. The WASOG Sarcoidosis Organ Assessment Instrument: An update of a previous clinical tool. Sarcoidosis Vasc Diffuse Lung Dis. 2014;31:19-27.

II. Kandolin R, Lehtonen J and Kupari M. Cardiac sarcoidosis and giant cell myocarditis as causes of atrioventricular block in young and middle-aged adults. Circ Arrhythm Electrophysiol. 20I I;4:303-9.

12. Nery PB, Beanlands RS, Nair GM, Green M, Yang J, McArdle BA, Davis $\mathrm{D}$, Ohira $\mathrm{H}$, Gollob $\mathrm{MH}$, Leung E, Healey JS and Birnie DH. Atrioventricular block as the initial manifestation of cardiac sarcoidosis in middle-aged adults. J Cardiovasc Electrophysiol. 20I4;25:875-88।.

13. Protonotarios A, Wicks E, Ashworth M, Stephenson E, Guttmann O, Savvatis K, Sekhri N, Mohiddin SA, Syrris P, Menezes L and Elliott P. Prevalence of (I8)F-fluorodeoxyglucose positron emission tomography abnormalities in patients with arrhythmogenic right ventricular cardiomyopathy. Int J Cardiol. 2019;284:99-104.

14. Mehta D, Mori N, Goldbarg SH, Lubitz S, Wisnivesky JP and Teirstein A. Primary prevention of sudden cardiac death in silent cardiac sarcoidosis: role of programmed ventricular stimulation. Circ Arrhythm Electrophysiol. 2011;4:43-8.

15. Zipse MM, Tzou WS, Schuller JL, Aleong RG, Varosy PD, Tompkins C, Borne RT, Tumolo AZ, Sandhu A, Kim D, Freeman AM, Weinberger HD, Maier LA, Sung RK, Nguyen DT and Sauer WH. Electrophysiologic testing for diagnostic evaluation and risk stratification in patients with suspected cardiac sarcoidosis with preserved left and right ventricular systolic function. J Cardiovasc Electrophysiol. 2019;30:1939-1948.

16. Kron J, Sauer W, Schuller J, Bogun F, Crawford T, Sarsam S, Rosenfeld L, Mitiku TY, Cooper JM, Mehta D, Greenspon AJ, Ortman M, Delurgio DB, Valadri R, Narasimhan C, Swapna N, Singh JP, Danik S, Markowitz SM, Almquist AK, Krahn AD, Wolfe LG, Feinstein $S$ and Ellenbogen KA. Efficacy and safety of implantable cardiac defibrillators for treatment of ventricular arrhythmias in patients with cardiac sarcoidosis. Europace. 2013;15:347-54.

17. G. Kowlgi RK, A. Chicos, D. Birnie, R. Meredith, A. Abbate, K. Ellenbogen, J. Kron, H. Syed, E. James, B. Houston, W. Rieter, T. Crawford, K. Wunderly, F. Bogun, J. Lehtonen. Infliximab Stabilizes Ejection Fraction and Reduces Ventricular Tachycardia in Refractory Cardiac Sarcoidosis. Heart Rhythm. 2019;16:S32.

18. Trivieri MG, Spagnolo P, Birnie D, Liu P, Drake W, Kovacic JC, Baughman R, Fayad ZA and Judson MA. Challenges in Cardiac and Pulmonary Sarcoidosis: JACC State-of-the-Art Review. J Am Coll Cardiol. 2020;76:1878-190I. 
19. Kumita S, Yoshinaga K, Miyagawa M, Momose M, Kiso K, Kasai T, Naya $M$ and Committee for diagnosis of cardiac sarcoidosis using I8F-Fdg Pet JSoNC. Recommendations for (I8)F-fluorodeoxyglucose positron emission tomography imaging for diagnosis of cardiac sarcoidosis-2018 update: Japanese Society of Nuclear Cardiology recommendations. J Nucl Cardiol. 2019;26:14|4-| 433.

20. Pender A, Sporn PHS, Russell SR, Passman R, Knight B and Chicos AB. High Risk of Adverse Outcomes in Cardiac Sarcoidosis is Associated with Failure to Suppress Myocardial Inflammation Assessed by FDG-PET Scanning. J Am Coll Cardiol. 2016;67:1823-1823.

21. Chareonthaitawee P, Beanlands RS, Chen W, Dorbala S, Miller EJ, Murthy VL, Birnie DH, Chen ES, Cooper LT, Tung RH, White ES, Borges-Neto S, Di Carli MF, Gropler RJ, Ruddy TD, Schindler
TH, Blankstein R and Name Of Collab G. Joint SNMMI-ASNC Expert Consensus Document on the Role of (I8)F-FDG PET/CT in Cardiac Sarcoid Detection and Therapy Monitoring. J Nucl Med. 2017;58:1341-1353.

22. K. Siontis PS, D. Muser, F. Marchlinski, K. Zeppenfeld, J. Hoogendoorn, C. Narasimhan, W. Sauer, M. Zipse, S. Kapa, V. Vedantham, D. Rosenthal, M. Robinson, K. Patton, F. Murgatroyd, A. Chicos, K. Soejima, H. Roukoz, F. Sacher, A. Bhan, J. Appelbaum, T. Dickfeld, P. Mankad, K. Ellenbogen, J. Kron, HM Kim, J. Froehlich, K. Eagle, F. Bogun, T. Crawford. Outcomes of ventricular tachycardia ablation in cardiac sarcoidosis: report from a multicenter, international registry. Heart Rhythm. 2019;16:S386. 